\title{
The Prevalence and Risk Factors of Paternal Sleep Problems Across the Perinatal Period in Hong Kong - a Longitudinal Study
}

\author{
Yee Woen Koh, Ph.D \\ the National University of Singapore \\ Antoinette Marie Lee, Ph.D \\ University of Hong Kong \\ Chui Yi Chan, Ph.D. \\ University of Hong Kong \\ Catherine So-Kum Tang, Ph.D \\ National University of Singapore \\ Jean Wei-Jun Yeung, Ph.D \\ National University of Singapore
}

\begin{abstract}
Background: The present study aimed to identify the prevalence of sleep problems among the fathers from the antenatal to postpartum period as well as their risk factors with an aim to develop strategies to assist the fathers during the transition period. Methods: A consecutive sample of 540 Chinese expectant fathers were recruited. Expectant fathers were administered a set of questionnaires at first presentation (12 weeks gestation), 36 weeks pregnancy and 6 weeks after childbirth. Sleep problems was assessed with the Pittsburgh Sleeping Quality Index (PSQI). Demographic and psychosocial risk factors were also assessed. Findings: An alarming $42.2 \%$, $57.5 \%$ and $70.3 \%$ of the fathers were identified to have sleep problems at early, late pregnancy and six week postpartum respectively. Hierarchical multiple regression analysis showed that poor self-esteem and work family conflict were significant risk factors for sleep problems at early pregnancy and late pregnancy respectively, even after controlling for confounders. Poor social support and work family conflict significantly predicted sleep problems at six week postpartum. Discussions: The prevalence of sleeping problems among the fathers during perinatal period was alarmingly high and warrants serious attention. Given the potential adverse impact of sleep problems on fathers' mental and physical health as well as caregiving abilities, findings from this study point to the need to equip fathers with psychosocial resources through perinatal psychoeducation, support group and counselling. Caution should be exercised in generalizing the results to fathers of other backgrounds as the sampling of the present study only include a certain area of Hong Kong.
\end{abstract}

Keywords: Paternal sleeping problems, risk factors 